\title{
Measuring Sensible Heat Flux with High Spatial Density
}

\author{
Alexander Bahr, Chris Evans, Alcherio Martinoli \\ Distributed Intelligent Systems and Algorithms Laboratory \\ School of Architecture, Civil and Environmental Engineering \\ Ecole Polytechnique Fédérale de Lausanne (EPFL) \\ Lausanne, Switzerland \\ firstname.lastnamedepfl.ch
}

\author{
Hendrik Huwald, Chad Higgins, Marc Parlange \\ Environmental Fluid Mechanics and Hydrology \\ chool of Architecture, Civil and Environmental Engineering \\ Ecole Polytechnique Fédérale de Lausanne (EPFL) \\ Lausanne, Switzerland \\ firstname.lastnamedepfl.ch
}

\begin{abstract}
Sensible heat flux is an essential quantity in the surface energy budget. Information about it is crucial for the derivation of models of air-surface interaction which in turn are needed for larger scale climate models. Sensible heat flux varies greatly across short distances and thus many sensors are required in order to obtain measurements of sufficient spatial density. The standard measurement method however requires expensive equipment which constrains the deployment of a large number of sensors. Our work implements a method relying only on the variance of the measured temperature to compute the sensible heat flux. We first verify the validity of this method and then modify SENSORSCOPE, a commercially available weather station, to capture the required data. The resulting setup is able to provide sensible heat flux measurements with high spatial density in near real time at negligible additional cost.
\end{abstract}

\section{INTRODUCTION}

Determining the surface energy balance at high spatial density is essential in order to establish boundary conditions for many hydrological and atmospheric boundary layer models. The energy exchange consists of four components: turbulent heat (sensible) flux, moisture (latent) heat flux, radiative flux and conductive flux. Of these four quantities, only the radiative component can be measured directly. For most of the radiative component a sensor is typically affordable enough to be deployed in sufficiently large numbers to obtain high spatial density. The sensible and latent heat fluxes are typically measured using the Eddy-Covariance (EC) method. For the sensible heat flux this method requires the vertical wind speed and the temperature fluctuations of a small sample volume at high frequency $(20 \mathrm{~Hz})$, both of which can be obtained using an (expensive) sonic anemometer. In addition, the latent heat flux requires a high frequency humidity measurement.

A typical sensor deployment for the study of boundary layer conditions usually consists of two groups of sensors. A larger number of sensor suites which only incorporate lowcost sensing modalities such as air temperature, humidity, wind speed and solar radiation in combination with a much smaller set of expensive sensors such as sonic anemometers [1]. In order to determine the sensible heat flux using only cheap sensors Albertson et al. describe the $\sigma_{T}$-method, which only relies on measuring the temperature at high frequency and computing the sensible heat flux from the temperature's mean and variance [2]. We leverage SENSORSCOPE [3], a commercially available wireless sensor network (WSN) technology recently developed for micro-climate research. The particular focus on micro-climate research makes SENSORSCOPE an ideal platform for measurements geared towards understanding air-surface interaction. By augmenting the existing system and relying on in-network computation we seamlessly add sensible heat flux to the existing set of measurements.

The remainder of this paper is organized as follows. Section II briefly outlines the $\sigma_{T}$-method, describes the SENSORSCOPE platform and the integration of the fast temperature sensor. In Section III, we first describe the experiment where the $\sigma_{T}$-method and the EC method are directly compared by using them to determine the sensible heat flux within the same sampling volume. After validating the $\sigma_{T}$-method we deployed a set of SENSORSCOPE stations augmented with the capability to take sensible heat flux measurements and collected sensible heat flux data with a number of stations simultaneously. In Section IV we conclude and discuss future directions.

\section{Materials AND MethodS}

\section{A. The Eddy-Covariance (EC) method}

The EC method (Eq. 1) is the reference against which the results from our deployment (which uses the $\sigma_{T}$-method) are compared against. The method is well established [4] and has been used in a large number of environments [5], [6]. It does however require high frequency measurements of the vertical wind velocity $w$ and temperature $T$. The high frequency data is usually obtained with a sonic anemometer and then used to compute the covariance (in time) between the vertical velocity and temperature fluctuations $\overline{w^{\prime} T^{\prime}}$ to obtain the sensible heat flux.

$$
\begin{aligned}
H_{E C}= & \rho c_{p} \overline{w^{\prime} T^{\prime}} \\
& \text { sensible heat flux }\left[\mathrm{W} / \mathrm{m}^{2}\right] \\
\rho= & 1.184 \text { density of air at } 25^{\circ} \mathrm{C}\left[\mathrm{kg} / \mathrm{m}^{3}\right] \\
c_{p}= & 1012 \text { spec. heat cap. of air at } 23^{\circ} \mathrm{C}[\mathrm{J} /(\mathrm{kg} \cdot \mathrm{K})]
\end{aligned}
$$




\section{B. The $\sigma_{T}$-method}

The $\sigma_{T}$-method obtains surface fluxes by applying the Monin-Obuknov similarity theory [7]. This technique requires steady state conditions over a homogeneous, flat land surface with zero-mean vertical fluid flow. The number of parameters which have to be measured can be further reduced by applying free convective scaling to the Monin-Obuknov similarity theory which requires the dominant forcing of atmospheric motions to arise from buoyancy. This occurs mainly during the daytime.

For this special case Albertson et al. [2] show that the sensible heat flux can be computed using Eq. 2, which only requires the first and second moment of the temperature measured with a sufficiently high sampling rate.

$$
\begin{aligned}
H_{\sigma_{T}}= & \sigma_{T}^{3 / 2} T_{a}^{-1 / 2} \rho c_{p} C_{1}^{-3 / 2}(\mathrm{kgz})^{1 / 2} \\
& \text { sensible heat flux }\left[\mathrm{W} / \mathrm{m}^{2}\right] \\
\sigma_{T}= & \text { variance of temperature } \\
\bar{T}_{a}= & \text { mean air temperature }[\mathrm{K}] \\
\rho= & 1.184 \text { density of air at } 25^{\circ} \mathrm{C} \\
& \text { and barometric pressure at } 0 \mathrm{~m} \text { a.s. } 1 .\left[\mathrm{kg} / \mathrm{m}^{3}\right] \\
c_{p}= & 1012 \text { spec. heat cap. of air at } 23^{\circ} \mathrm{C}[\mathrm{J} /(\mathrm{kg} \cdot \mathrm{K})] \\
C_{1}= & 0.97 \text { universal constant, see }[2] \\
k= & 0.4 \text { von Karman constant } \\
g= & 9.81 \text { gravitational acceleration }\left[\mathrm{m} / \mathrm{s}^{2}\right] \\
z= & 1.2 \text { sensor height over ground }[\mathrm{m}]
\end{aligned}
$$

For details of Eq. 2 please refer to [2], [8].

\section{The SEnSORSCOPE platform}

The SENSORSCOPE platform [9] is a tool which has been specifically developed for micro-climate research. The overall system consists of a group of wirelessly networked meteorological stations where each station is affordable enough to allow for a deployment of a large $(10-50+)$ number of stations. The stations are typically deployed over a comparably small area $\left(1-10\right.$ stations per $\left.\mathrm{km}^{2}\right)$ and thus provide meteorological data with high spatial density.

Each station can be equipped with a variety of meteorological sensors such as temperature, humidity, wind speed/direction, precipitation, solar radiation, soil moisture and surface temperature. The sensors can be multiplexed and the modular setup lets the user swap out sensor modules as needed. All sensors of a station are typically attached to a single aluminum pole. For easy reconfiguration of individual stations each sensor module consist of the sensor itself and a dedicated processing unit. The processing unit contains a microcontroller (Texas Instruments MSP430 series) which samples the sensor and provides the data on a serial bus. The data bus allows for simple daisy-chaining of multiple sensor modules. This chain of sensors is attached to a data logger unit which controls the entire station. The data logger contains a similar microcontroller as the sensor unit and a short range (ca.
$1 \mathrm{~km}$ ) transceiver operating at $900 \mathrm{MHz}$. After being powered up, the data logger uses the short range transceiver to establish a network by listening for beacons from nearby stations and thereby building a routing. At least one station in the network is outfitted with a cell phone-based data connection (GPRS or UMTS) and serves as network sink. All stations are connected to this station via a single or multi-hop link. Fig. 1 shows the midsection of a SENSORSCOPE station deployed during our experiment. The software operating on the data logger as well as in each individual sensor module is implemented in Tiny OS [10]. Each station is powered by a battery-buffered solar cell attached directly to the data logger.

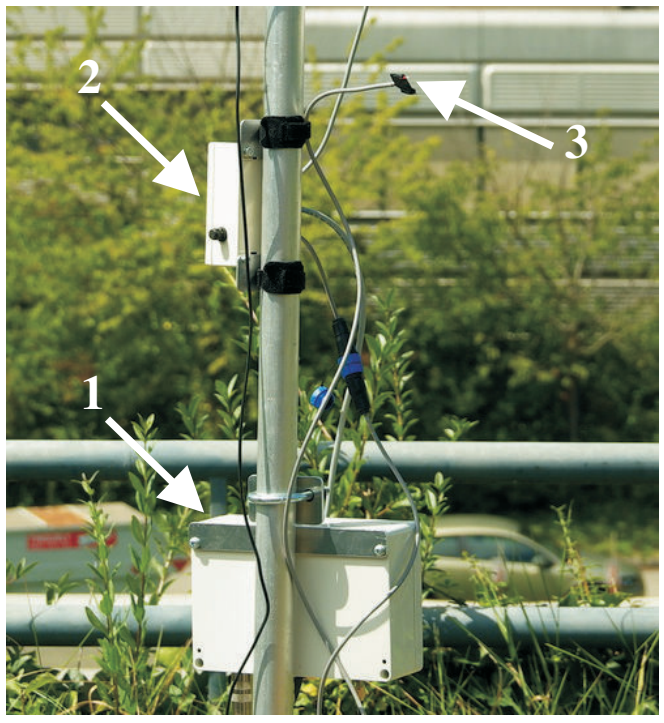

Fig. 1. A SENSORSCOPE station consisting of a data logger (1), a sensor module (processing unit and sensor) (2) and with a fast NTC (3).

The entire network usually operates on a fixed schedule where all stations wake up after a predefined interval $\Delta \mathrm{t}_{q}$ using a clock which is synchronized to the sink's clock. After waking up, each station transmits data collected in the interim over the short range radio towards the sink.

\section{Sensor}

To measure the temperature we decided to use a very small Negative Temperature Coefficient thermistor (NTC) from Honeywell's 111 series. The key specifications are listed in Table I. The small size of the sensor leads to a very short time constant $\tau$ which allows temperature measurements with sufficiently high frequency.

\section{E. Sensor integration}

Each sensor on a SENSORSCOPE station has a dedicated microcontroller. As a sensor only needs a small subset of the peripherals offered by the microcontroller we integrated our fast NTC by attaching it to an unused analog-to-digital converter (ADC) of a standard temperature/humidity sensor provided by SENSORSCOPE (Fig. 2). The supply voltage for the NTC is provided by a high precision $2.5 \mathrm{~V}$ reference which is part of the sensor module. Due to the power dissipated as a 


$\begin{array}{ll}\text { Resistance at } 25^{\circ} \mathrm{C} \mathrm{R} 0 & 2000 \Omega \\ \text { Operating Temperature } & -60^{\circ} \mathrm{C} \text { to } 300^{\circ} \mathrm{C} \\ \text { Beta } & 3068 \\ \text { Diameter } d & 0.36 \mathrm{~mm} \\ \text { Time Constant in Air } \tau & 0.5 \mathrm{~s} \\ \text { Dissipation Constant } K & 0.1 \mathrm{~mW} /{ }^{\circ} \mathrm{C}\end{array}$

TABLE I

Product SPECIFICATIONS OF Negative TEMPERATURE COEFFicient THERMISTOR (NTC)

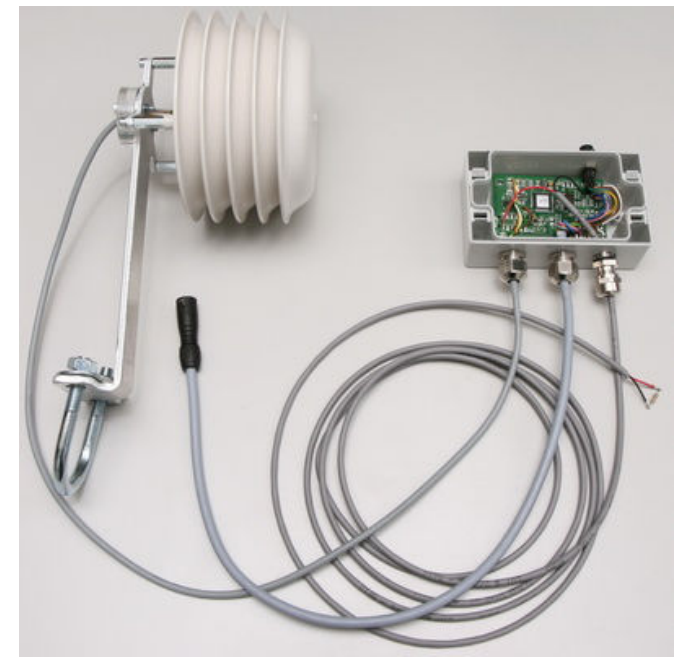

Fig. 2. A modified sensor module from a SENSORSCOPE station: the original temperature/humidity sensor (left) attached to the leftmost port of the sensor module, the connector for the sensor bus (center port), cable with a fast NTC, here substituted with a fixed resistor (rightmost port)

result of a current flowing through it during measurements, the NTC heats up. This self-heating temperature $\Delta T$ is directly related to the current $I$ flowing through the NTC, the dissipation constant $K$ and the diameter $d$ of the NTC as follows

$$
\begin{aligned}
\Delta T & \propto I^{2} \\
\Delta T & \propto \frac{1}{K} \\
K & \propto d^{2}
\end{aligned}
$$

When averaging temperatures measured with the NTC over long time periods, $\Delta T$ can be considered as a constant offset which one can easily account for. For short time intervals considered in our application however the NTC acts like a high frequency hot-wire anemometer and the turbulent wind flows around the sensor greatly increase $\sigma_{T}$. Due to the small size $d$ of our NTC this effect is very pronounced and thus $I$ needs to be as small as possible.

In addition to attaching the sensor to the microcontroller, the original software of the temperature/humidity sensor-module also required modification. While all sensor modules in a typical SENSORSCOPE operation are continuously powered through the bus, the microcontroller in each sensor module usually remains in a sleep state so as to conserve power and only becomes active when the sensor is queried. Our method however requires continuous measurements. We thus modified the software such that the microcontroller does not enter the sleep state and samples the NTC at $10 \mathrm{~Hz}$. The available memory on the microcontroller is far too small to store all samples and then compute the temperature's mean $\bar{T}$ and variance $\sigma_{T}$ of all collected samples. We thus use Algorithm 1 presented in [11] which continuously updates $\bar{T}$ and $\sigma_{T}$. After each time interval $\Delta \mathrm{t}_{q}$ the sensor is queried by the data logger. It then reports the actual value for $\bar{T}$ and $\sigma_{T}$ which are then reset.

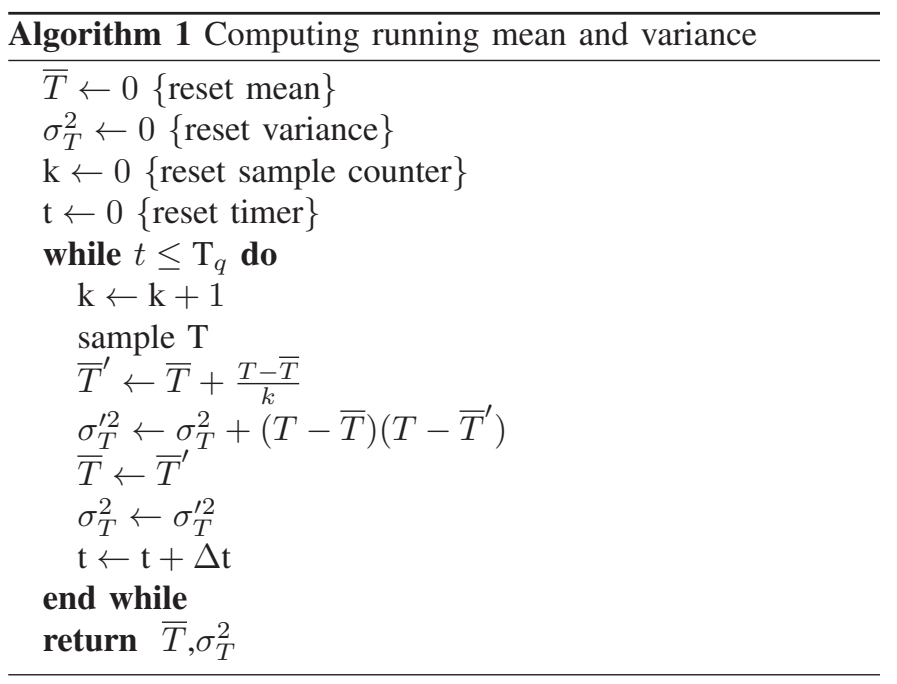

When the sensor module is queried by the data logger after $\Delta \mathrm{t}_{q}, \bar{T}$ and $\sigma_{T}$ are sent across the data bus.

\section{EXPERIMENTS}

In order to verify whether the method described in [2] using $\sigma_{T}$ does deliver results comparable to the standard EC method, we first set up an experiment where both methods were used to measure the sensible heat flux within the same volume. After this experiment confirmed the validity of the $\sigma_{T}$-method we deployed a network of seven SENSORSCOPE stations where five were outfitted with an NTC.

\section{A. Reference experiment}

The first experiment provided a direct comparison between the EC and the $\sigma_{T}$-method. An ultrasonic anemometer (Campbell Scientific CSAT3) capable of measuring the 3D wind velocity vector and the temperature of the air within the sample volume was mounted on a thin but rigid structure $3 \mathrm{~m}$ above a flat, open, grass-covered surface. Fig. 3 shows the entire setup and Fig. 4 shows a close-up of the sample volume which also contains a fine wire thermocouple (Type E, Campbell Scientific FW3) and a thin wire thermocouple (Type T, OmegaNewport, $2 \mathrm{~mm}$ twisted tip) in addition to the NTC.

\section{B. Sensorscope deployment}

Data from all sensors were collected at $20 \mathrm{~Hz}$ and stored using a Campbell Scientific CR5000 data logger. Data from the sonic anemometer were then processed using the EC 


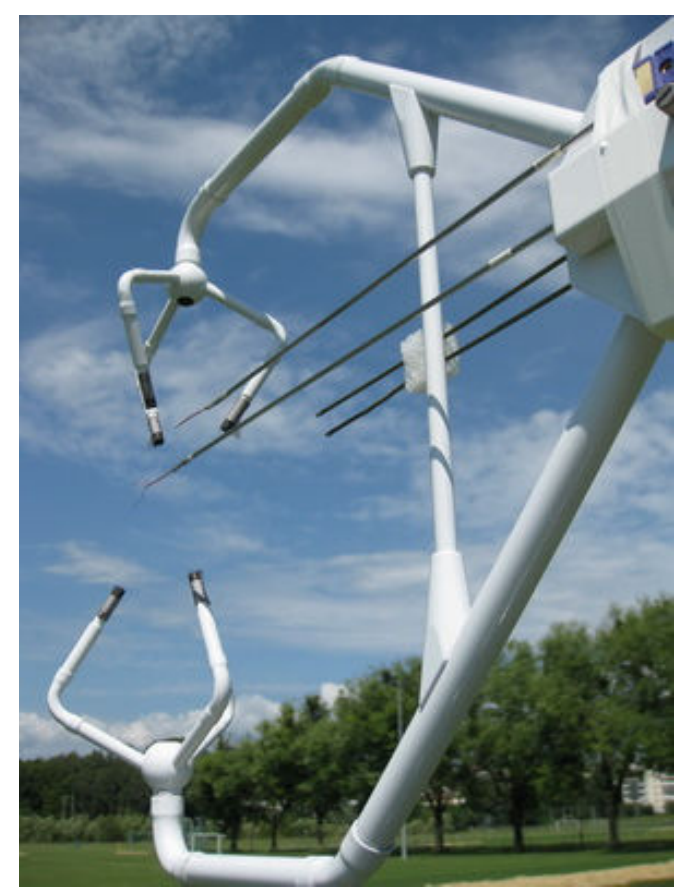

Fig. 3. Setup for direct comparison between the $\sigma_{T}$ and the EC method

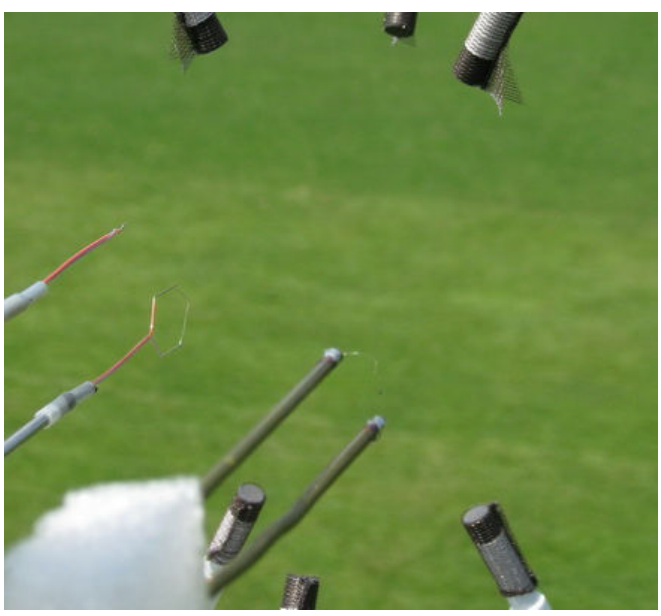

Fig. 4. Close-up of reference setup. top and bottom: sonic anemometer; center, from left to right: thin wire, fine wire thermo couple, NTC

method and data from the NTC were processed using the $\sigma_{T}$-method. Data from the fine wire thermocouple as well as the thin wire thermocouple was also used to independently compute the sensible heat flux using the $\sigma_{T}$-method. While only an NTC was used in the later deployment using multiple stations, data from the thermocouples was used to assess their usability (frequency response, accuracy, ruggedness) for the $\sigma_{T}$-approach. The results in Fig. 5 show a strong similarity for a period of several days. Details about this experiment and in particular the effect of varying the sampling rates, the sensor and averaging period as well as requirements for the sensors involved can be found in [8].

The sensor network deployment consisted of seven SEN-

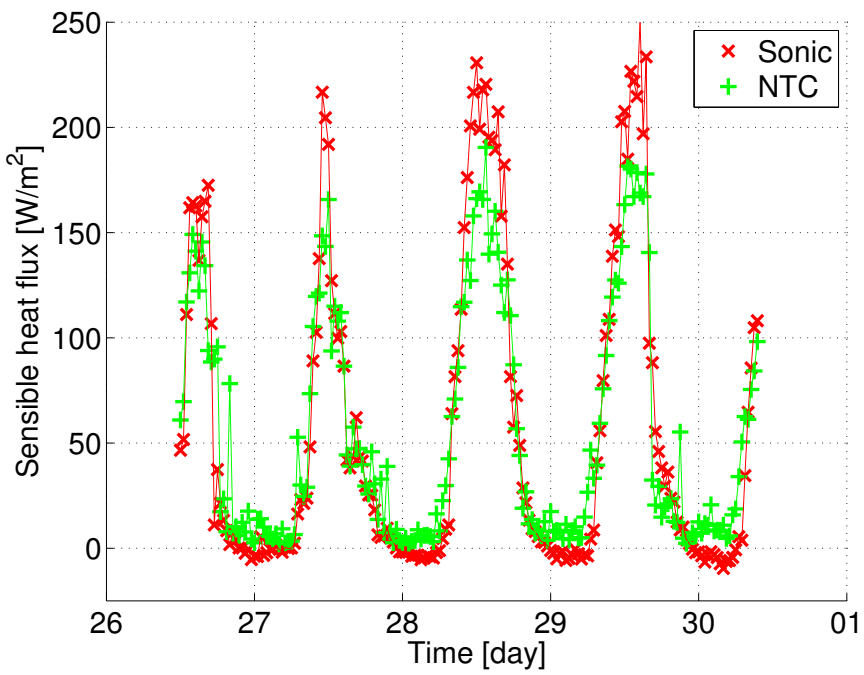

Fig. 5. Comparison between sensible heat flux determined by EC method (red) and $\sigma_{T}$-method (green)

SORSCOPE stations out of which one was serving solely as a sink and did not have any sensors attached. Of the remaining stations five were outfitted with an NTC as described in Section II. As the NTCs were seamlessly integrated in the existing SENSORSCOPE hardware and software architecture, no particular steps were required during the setup. The stations were installed at various places around our laboratory's building. Measurements were taken over a period of several weeks, however a period of only one week was used for data analysis. During these several weeks, none of the NTCs broke despite their small size and exposed position. As established in the previous experiment, the NTCs did not require any calibration before or any maintenance during the experiment. In addition to the NTC, each station was outfitted with an enclosed temperature/humidity sensor (Sensirion SHT75) as shown in Fig. 2. Each station's data logger queried all sensors at an interval of $\mathrm{T}_{q}=60 \mathrm{~s}$ and forwarded the data to the sink through the $900 \mathrm{MHz}$ network. Higgins et al. established in [8] that the ideal length for the time window over which the data should be averaged is $5-10$ minutes and the acquired data was thus downsampled to 5 minute intervals. Fig. 5 shows the sensible heat flux as computed from the data obtained from three of the five stations. The computed heat fluxes match reasonably well and differences can be explained through the different locations of the stations. We also validated the proper operation of the NTC through a comparison between the mean temperature of the NTC collected during a five minute observation interval and the instantaneous measurement of the SHT75 when the sensors were queried (Fig. 6). As for the differences in Fig. 6 please note that the NTC's temperature was affected by self heating and the SHT75 by radiative heating of its enclosure.

Most WSN technology, including SENSORSCOPE, reduces power consumption by minimizing on-time in order to satisfy the strict power requirements imposed by 24 hour, all- 


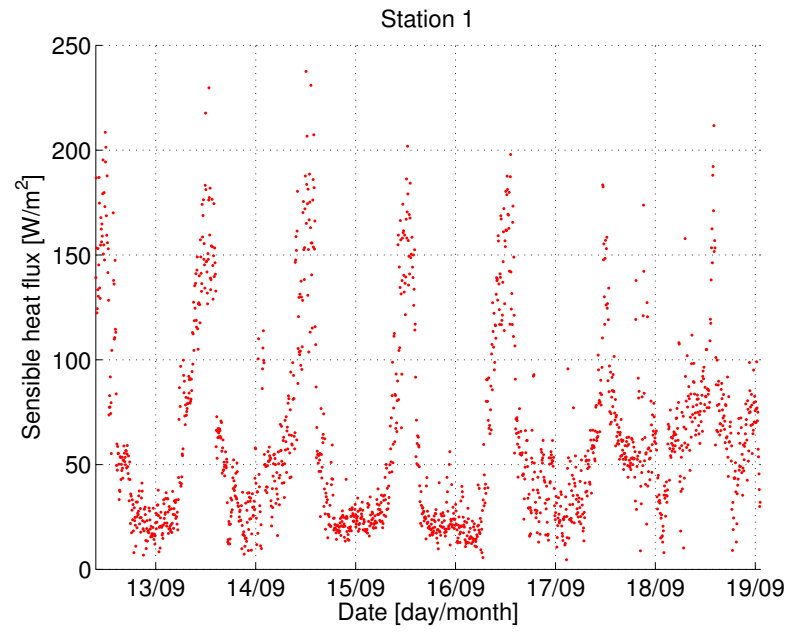

(a)

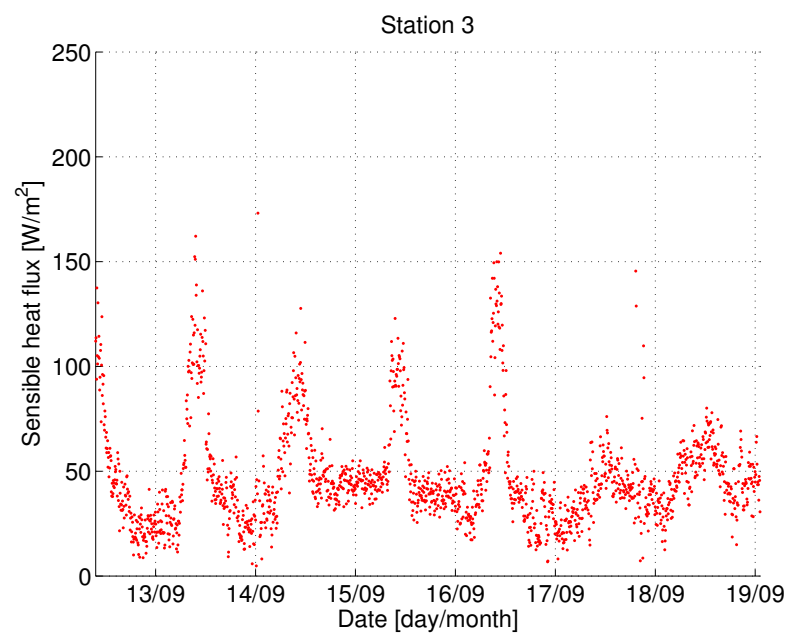

(b)

weather operation with only a moderately sized solar cell. As the $\sigma_{T}$-method requires the sensor and the processor to be continuously active, it was particularly important to keep the power consumption low. Adding the NTC and maintaining the corresponding MSP430 microcontroller in an active state increased the power consumption by $5 \mathrm{~mW}$ and we established that we can reduce it further by significantly cutting the NTC excitation current. The installed solar cell (size: $1 \mathrm{dm}^{2}$ ) proved adequate.

The total price of all additional components for integrating sensible heat flux measurement is about $\$ 30$. As a result our sensor/method is affordable enough to become standard on all stations during a deployment.

\section{CONCLUSiOnS AND Future Work}

The goal of our work is to obtain sensible heat flux, a parameter with high spatial variability which is important in investigating earth's surface energy budget, with high spatial density. We leverage existing hardware for micro-climate research as the sensing capabilities are provided as a plug-in

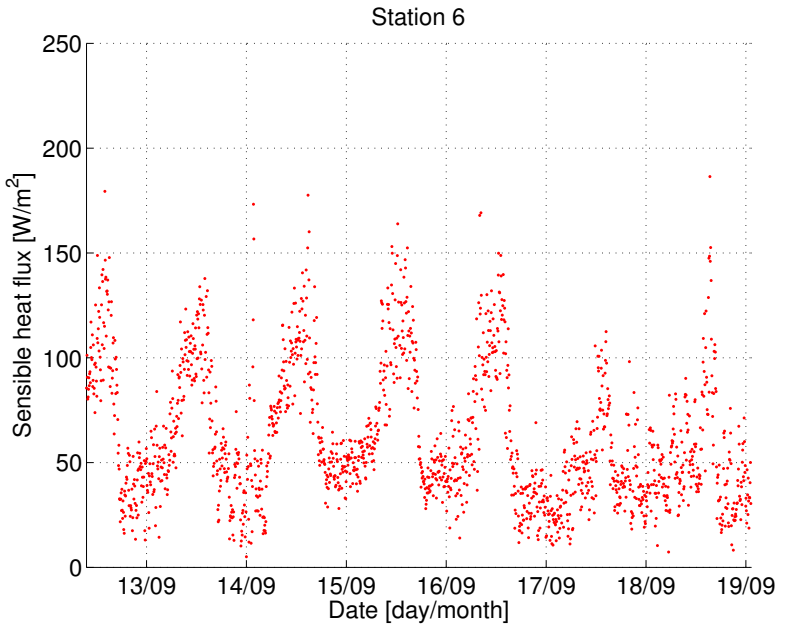

(c)

Fig. 5. Sensible heat flux data from three of the five SENSORSCOPE stations obtained over the period of one week (window length: 5 minutes)

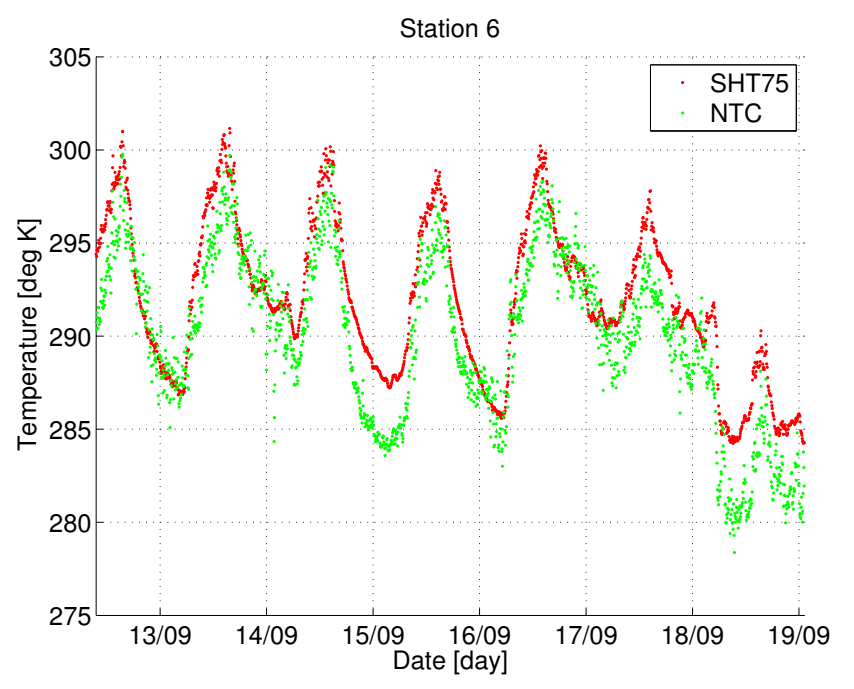

Fig. 6. Temperature measurements from the Sensirion SHT75 (green) and averaging the NTC data over 5 minute intervals

module for the SENSORSCOPE system. By selecting the $\sigma_{T^{-}}$ method and relying on in-sensor preprocessing we provide a method which is suitable for the power and data transfer constraints imposed by WSNs.

The performance of a single setup was first compared against a well established reference. In a subsequent deployment of five stations we showed that the equipment used in this approach is robust enough to resist meteorological conditions over an entire summer season yet cost-effective enough that existing sensing stations can be upgraded to include sensible heat flux measurements at negligible costs.

In upcoming experiments our upgraded stations will be part of a large scale deployment which will allow us to assess the long time reliability of our method. A number of sonic anemometers used in this deployment will also provide 
further opportunities to cross-verify the applicability of the $\sigma_{T}$-method with the EC-method.

\section{ACKNOWLEDGEMENTS}

This work was partially funded by "The Swiss Experiment" of the Competence Center Environment and Sustainability of the ETH Domain (CCES), and by the Swiss National Science Foundations Stabilization Measures Program, associated with the project Tamperproof Monitoring Solution for Weather Risk Management managed by the National Center of Competence in Research in Mobile Information and Communication Systems (NCCR-MICS).

\section{REFERENCES}

[1] S. Simoni, S. Padoan, D. Nadeau, M. Diebold, A. Porporato, G. Barrenetxea, F. Ingelrest, M. Vetterli, , and M. B. Parlange, "Hydrologic response of an alpine watershed: Application of a meteorological wireless sensor network," Water Resources Research, accepted.

[2] J. D. Albertson, M. B. Parlange, G. G. Katul, C.-R. Chu, H. Stricker, and S. Tyler, "Sensible heat flux from arid regions: A simple flux-variance method," Water Resources Research, vol. 31, no. 4, pp. 969-973, 1995.

[3] "http://www. sensorscope.ch," 2011.

[4] Baldocchi, "FLUXNET: A New Tool to Study the Temporal and Spatial Variability of Ecosystem-Scale Carbon Dioxide, Water Vopor, and Energy Flux Densities," Bulletin of the American Meteorological Society, vol. 82, no. 11, pp. 2415-2434+, 2001.

[5] H. Soegaard, N. Jensen, E. Boegh, C. Hasager, K. Schelde, and A. T. A., "Carbon dioxide exchange over agricultural landscape using eddy correlation and footprint modelling," Agricultural and Forest Meteorology, vol. 114, no. 3, pp. 153-173, 2003.

[6] M. L. Reba, T. E. Link, D. Marks, and J. Pomeroy, "An assessment of corrections for eddy covariance measured turbulent fluxes over snow in mountain environments," Water Resources Research, vol. 45, 2009.

[7] A. S. Monin and A. M. Obukhov, "Basic laws of turbulent mixing in the surface layer of the atmosphere," Contrib Geophys Inst Acad Sci USSR151, vol. 24, no. 151, pp. 163-187, 1959.

[8] C. Higgins, H. Huwald, A. Bahr, A. Martinoli, and M. Parlange, "Sensible heat flux from wireless environmental sensor networks," Water Resources Research, submitted.

[9] G. Barrenetxea, F. Ingelrest, G. Schaefer, M. Vetterli, O. Couach, and M. Parlange, "Sensorscope: Out-of-the-box environmental monitoring," in Proc. Int. Conf. Information Processing in Sensor Networks IPSN '08, 2008, pp. 332-343.

[10] J. Hill, R. Szewczyk, A. Woo, S. Hollar, D. Culler, and K. Pister, "System architecture directions for networked sensors," in Proceedings of the 9th International Conference on Architectural Support for Programming Languages and Operating Systems, Cambridge, MA, USA, November 2000, pp. 93-104.

[11] B. P. Welford, "Note on a method for calculating corrected sums of squares and products," Technometrics, vol. 4, no. 3, pp. pp. 419-420, 1962. [Online]. Available: http://www.jstor.org/stable/1266577 